\title{
Chemische Untersuchung eines antiken Wasserleitungs-Kittes
}

\author{
von
}

Friedrich Dörner.

Aus dem chemischen Laboratorium der k. k. technischen Hochschule in Graz.

(Vorgelegt in der Sitzung am 3. Februar 1899.)

Zu Beginn des vorigen Jahres wurden dem chemischen Laboratorium der Grazer technischen Hochschule von Herrn Prof. Dr. Ph. Forchheimer, der eben von einer Studienreise nach Kleinasien zurückgekehrt war, Kittproben vorgelegt, deren Prüfung mir Herr Prof. Emich übertrug. Da die vorhandenen Mengen zu der wünschenswerthen genauen Untersuchung nicht genügten, veranlasste Herr Prof. Forchheimer die Einsendung weiteren Materiales. Einen Theil desselben hatte Herr Dr. Heberdey - damals in Ajasoluk - die Freundlichkeit zu übersenden, welcher ihn den Thonleitungen im Südwesten der Agora und den kleinen Leitungen des Theaters in Ephesus entnahm; ein anderer Theil, welchen Herr Prof. Weber in Smyrna gütigst zur Verfügung stellte, stammte aus einer Steinrohrleitung bei Smyrna, und zwar aus Lochsteinen, welche gelegentlich des Bahnbaues verschleppt worden waren.

Das Material stellt eine hellgraue, ziemlich feste Masse von ungleichartiger Structur dar. Man kann an den Bruchflächen geschichtete und wieder ganz gleichartige Theile unterscheiden, auch fallen stecknadelkopfgrosse Sandkörner auf; Reste von Faserstoffen konnten nicht gefunden werden. Schon eine oberflächliche Untersuchung durch Lösen eines Stückes 
in verdünnter Salzsäure zeigte das Vorhandensein von organischer Substanz. Von derselben geht ein kleiner Theil in die Lösung über, ihr eine gelbliche Färbung ertheilend, während der grösste Theil ungelöst bleibt. Die Untersuchung zeigte ferner die Anwesenheit von Calciumcarbonat; auch wurden ausserdem noch Wasser, Kieselsäure, Eisen, Aluminium, Magnesium, Natrium in kleinen Mengen gefunden. Ammoniak, Salpetersäure und Phosphorsäure konnten nicht nachgewiesen werden. Die quantitative Analyse, ausgeführt mit einem Stück, welches aus der Leitung im Südwesten der Agora in Ephesus stammte, ergab:

I.

Calciumoxyd ...........52 $65 \%$

Aluminium- und Eisenoxyd ... $0 \cdot 27$

Magnesiumoxyd.......... $0 \cdot 7$

Natriumoxyd ............ 0.5

Kieselsäure............ 1.7

Kohlensäure ...........40 33

Wasser.............. 0.98
II.

$52 \cdot 51 \%$

$0 \cdot 22$

$0 \cdot 71$

$0 \cdot 59$

$1 \cdot 61$

$40 \cdot 57$

$0 \cdot 9$

Der Rest von circa $2 \cdot 8 \%$ ist organische Substanz. Eine vergleichende Bestimmung des Calciumoxydgehaltes der beiden anderen Kittproben führte zu folgenden Resultaten:

I.

Aus den Lochsteinen der Leitung von

Smyrna................. 52.93\%

Aus den Leitungen des Theaters von

Ephesus ................. $52 \cdot 31$
II.

$52 \cdot 88 \%$

$52 \cdot 46$

Das Material war sonach, obwohl von verschiedenen Plätzen stammend, der Zusammensetzung nach im Wesentlichen dasselbe, weshalb ich auch bei der nun folgenden Untersuchung der organischen Substanz die ganze Menge (circa $5 \mathrm{~kg}$ ) ohne Rücksicht auf Fundort zusammen verarbeitete. Hiebei leitete mich auch der Umstand, dass die Proben so ziemlich aus derselben Zeitperiode herrühren dürften. Es soll nämlich das Theater von Ephesus aus der Mitte des zweiten und die Agora aus denı Ende des dritten Jahrhunderts n. Chr. 
stammen. Bezüglich des Alters der Wasserleitungs-Anlage in Smyrna ist allerdings, wie es scheint, gegenwärtig nichts bekannt. Vielleicht stammt sie aus der Zeit der Neuerbauung, der im Jahre 630 v. Chr. durch den Lyderkönig Alyattes dem Erdboden gleichgemachten alten Stadt an der jetzigen Stelle, also etwa aus dem Anfange des zweiten Jahrhunderts v. Chr.; vielleicht wurde sie zur Zeit der Wiederherstellung des im Jahre 178 und 180 n. Chr. durch Erdbeben fast gänzlich zerstörten Ortes unter Marc Aure1, d. i. gegen Ende des zweiten Jahrhunderts n. Chr. erbaut.

Um die organischen Bestandtheile zu isoliren, wurde das gesammte Material mit verdünnter Salzsäure behandelt, filtrirt, der Rückstand getrocknet und mit Äther extrahirt. Der ätherlösliche Theil (circa $45 \mathrm{~g}$ ) stellte eine tiefbraune, butterartige, theilweise krystallinische Masse dar; der ätherunlösliche Theil bestand aus Sand, dem noch etwas organische Substanz beigemengt war.

\section{A. Ätherlöslicher Theil.}

Um eine Trennung der festen und flüssigen Antheile desselben zu erzielen, wurde die Masse etwa zehnmal aus heissem Alkohol umkrystallisirt, bis einerseits weisse, aschenfreie Krystalle (circa $15 \mathrm{~g}$ ), anderseits eine syrupöse, braune, ebenfalls aschenfreie Flüssigkeit (circa $10 \mathrm{~g}$ ) resultirte, die keine Neigung zur Krystallbildung mehr zeigte. Beide Theile waren stickstofffrei.

Derkrystallinische Theil besass einen Schmelzpunkt von $67^{\circ} \mathrm{C}$. und einen Erstarrungspunkt von $62 \cdot 3^{\circ} \mathrm{C}$., worin auch ein Umkrystallisiren keine Änderung hervorbrachte. Die Elementaranalyse ergab folgendes Resultat:

$$
\begin{aligned}
& \text { I. II. III. } \\
& \text { C } \ldots \ldots 75 \cdot 73 \% \quad 75 \cdot 8 \% \quad 75 \cdot 94 \% \\
& \begin{array}{llll}
\mathrm{H} \ldots \ldots 12 \cdot 55 & 12 \cdot 65 & 12 \cdot 71
\end{array}
\end{aligned}
$$

Die Substanz zeigte Säurecharakter und benöthigte zur Neutralisation

I.

II.

$$
\text { KHO . . . 2 20.05\% } 19 \cdot 89 \%
$$


Berücksichtigt man, dass für Palmitin-, respective Stearnsäure die bezüglichen Werthe wie folgt gefunden wurden:

\begin{tabular}{|c|c|c|c|c|}
\hline & C & $\mathrm{H}$ & KHO & $\begin{array}{l}\text { Schmelz- } \\
\text { punkt }\end{array}$ \\
\hline Palmitinsäure.. & $75 \cdot 0 \%$ & $12 \cdot 5 \%$ & $21 \cdot 05 \%$ & $62^{\circ} \mathrm{C}$ \\
\hline Stearinsäure .. & $76 \cdot 05$ & $12 \cdot 69$ & $19 \cdot 86$ & 69 \\
\hline
\end{tabular}

und berücksichtigt man ferner, dass ein Gemenge von 90 Theilen Stearinsäure und 10 Theilen Palmitinsäure bei $67 \cdot 2^{\circ} \mathrm{C}$. schmilzt und bei $62.5^{\circ} \mathrm{C}$. erstarrt, ${ }^{1}$ so kann mit aller Berechtigung gesagt werden, dass die vorliegende Substanz ein Gemisch dieser Säuren in annähernd dem erwähnten Verhältnisse darstellte.

Der flüssige Theil zeigte folgende Zusammensetzung:

I.

II.

$$
\begin{array}{ll}
\text { C } \ldots \ldots 68 \cdot 91 \% & 69 \cdot 08 \% \\
\text { H. . . } \ldots 10.57 & 10 \cdot 71
\end{array}
$$

Zur Neutralisation waren nöthig:

II.

$$
\mathrm{KHO} \ldots \ldots 20 \cdot 21 \% \quad 20 \cdot 5 \%
$$

Da die Substanz nur $23 \cdot 54 \%$ Jod addirte, so war die Wahrscheinlichkeit vorhanden, dass ein Gemenge von gesättigten und ungesättigten Verbindungen vorlag. Es wurde versucht, durch Destillation mit Wasserdampf einen einheitlichen Körper $\mathrm{zu}$ isoliren. Die auf diese Art (aus circa $6 \mathrm{~g}$ ) erhaltene Substanz (circa $1 / 2 g$ ) war lichter gefärbt, leichtflüssiger und zeigte folgende Zusammensetzung:

I.

II.

$$
\begin{array}{ll}
\text { C.....71.74\% } & 72 \cdot 0 \% \\
\text { H. .... } \% 10 \cdot 97 & 11 \cdot 19
\end{array}
$$

1 Heintz, Annalen der Chemie, 92, 1854, S. 295. 
Zur Neutralisation waren $18.99 \%$ KHO nöthig; die Jodzahl wurde mit $28 \cdot 17$ und 28.01 bestimmt, woraus geschlossen wurde, dass man es noch immer nicht mit einem Individuum $z u$ thun hatte, da von jenen bekannten ungesättigten Säuren, welche hier in erster Linie in Betracht kommen können, keine weniger als $75 \%$ Jod addirt. Eine weitere Untersuchung musste wegen Materialmangel unterbleiben. Es wurde nur noch festgestellt, dass weder die ursprüngliche flüssige Substanz, noch der durch Destillation mit Wasserdampf erhaltene Theil die Elaïdinreaction gäbe.

\section{B. Ätherunlöslicher Theil.}

Der von der Ätherextraction zurückgebliebenen Masse konnte weder durch Alkohol, noch durch Schwefelkohlenstoff, Benzol oder Petroläther etwas entzogen werden. In Wasser war der organische Körper allerdings ein wenig mit gelber Farbe löslich, und zwar umso leichter, je weniger dieses an Säuren und Salzen enthielt. Auf diesen Umstand war auch die geringe Lösung von organischer Substanz beim Behandeln des Ausgangsmateriales mit verdünnter Salzsäure zurückzuführen. Hier mag zugleich erwähnt werden, dass in dieser Lösung: kein Glycerin nachgewiesen werden konnte. Der Körper löste sich in Lauge, Soda, Ammoniak und Ammoniumcarbonat leicht mit brauner Farbe auf und konnte aus dieser Lösung durch Säuren in Form tiefbrauner Flocken wieder abgeschieden werden. Dieses Verhalten wies darauf hin, dass man es mit einer in die Classe der Huminverbindungen gehörigen Sub$\operatorname{stanz} \mathrm{zu}$ thun hatte. Um dieselbe möglichst rein zu erhalten, wurde sie oftmals in kohlensaurem Ammoniak gelöst, durch Salzsäure gefällt und mit säurehältigem Wasser gewaschen, bis ein Product (circa $12 \mathrm{~g}$ ) erhalten wurde, das, in Mengen von circa $0.3 \mathrm{~g}$ verbrannt, keine wägbare Menge Asche hinterliess. Der Körper war stickstofffrei und konnte bei $110^{\circ} \mathrm{C}$. bis $\mathrm{zu}$ constantem Gewicht getrocknet werden, ohne dass hiebei Silbernitratlösung reducirende Dämpfe abgegeben worden wären. ${ }^{1}$

1 M. Conrad und M. Guthzeit, B. 19, 1886, S. 2844. 
Die Elementaranalyse ergab folgendes Resultat:

I.

$$
\begin{aligned}
& \mathrm{C} \ldots \ldots \ldots 61 \cdot 78 \% \\
& \mathrm{H}=\ldots \ldots \ldots 7 \cdot 41
\end{aligned}
$$

II.

Von diesen Werthen liegt der für den Kohlenstoff gefundene innerhalb der für Huminverbindungen bis nun erhaltenen Grenzzahlen $(48 \cdot 8-76 \cdot 7 \%$ ), während der Wasserstoffgehalt ein merklich höherer ist als der der bis jetzt bekannten Verbindungen dieser Art $(2 \cdot 2-5 \cdot 1 \%)$. Trotz des letzteren Umstandes möchte ich den Körper wegen seiner physikalischen und chemischen Eigenschaften in diese Classe einreihen, umsomehr, als für Verbindungen dieser Gruppe noch keine strenge Charakteristik bezüglich chemischer Zusammensetzung geltend gemacht werden konnte.

Die Resultate dieser Untersuchung zusammenfassend, erkennen wir, dass aller Wahrscheinlichkeit nach in dem Kittmaterial der von Vitruvius, respective Plinius erwähnte Ölkitt, welcher durch Zusammenreiben von "lebendem « Kalk und Öl erhalten wurde, vorlag. ${ }^{1}$ Über die Natur des verwendeten Öles sprechen sich die Genannten nicht näher aus, doch ist nach einer gütigen Mittheilung des Herrn Prof. Dr. C. B. Hofmann unter »Öl « schlechtweg immer Olivenöl zu verstehen. Auch wurde noch bis gegen die Mitte dieses Jahrhunderts in Süditalien und Sicilien zur Herstellung von Kitt Olivenöl und Kalk; allerdings unter Zumischung von Faserstoffen, verwendet. ${ }^{2} \mathrm{Da}$ ich aber nicht im Vorhinein die Möglichkeit der Anwendung eines anderen viel verwendeten Öles - des Leinöles -, welches noch heute gemischt mit gelöschtem Kalk und Baumwolle zur Dichtung von Wasserleitungsröhren von den Türken benützt wird, ${ }^{3}$ für ausgeschlossen halten konnte und ein Aufschluss über die Art des angewendeten

1 Vitruvius, Zehn Bücher über Architectur. Ausgabe von Reber. 8. Buch, S. 252. - Plinius, LXXXI, c. 6.

2 Spataro, Les luyaux pour conduites d'eau. La technologie sanitaire, $1897 / 98$, p. 374 .

3 Forchheimer, Zeitschrift deutscher Ingenieure, 1891, S. 898 una 1033. 
Kalkes - ob gebrannter oder luftzerfallener - nicht ohne Interesse schien, so stellte ich eine Reihe von Versuchen an, bei welchen ich Oliven-, respective Leinöl einerseits und gebrannten, respective luftzerfallenen Kalk anderseits in verschiedenen Verhältnissen mischte und das Verhalten dieser Mischungen beobachtete. Hiebei ergab sich Folgendes: Die Mischungen von beiden Ölarten mit gebranntem Kalk begannen, wenn sie frei an der Luft liegen blieben, noch bevor sie ganz fest geworden, Risse und Sprünge zu bekommen und waren nach 14 Tagen völlig zerfallen; wurden sie aber in offenen Gefässen aufbewahrt, so waren sie nach Verlauf von zwei Monaten noch nicht erstarrt. Die Mischungen von beiden Ölarten mit luftzerfallenem Kalk dagegen wurden nach einiger Zeit ganz fest, und zwar erhärtete das Gemisch von zwei Theilen luftzerfallenem Kalk und einem Theil Olivenöl am schnellsten.

Es ist also anzunehmen, dass sich die Alten einer ähnlichen Mischung bedient haben und dass die grosse Differenz $z$ wischen den Mengen der angewendeten organischen Substanz - circa $30 \%$ - und der vorgefundenen - circa $21 / 2 \%$ - der vielhundertjährigen Einwirkung der Atmosphärilien, also einem Verwitterungs- und Auslaugungsprocess zuzuschreiben ist, bei welchem die Ölsäure gänzlich verändert und das Glycerin völlig ausgewaschen wurde. $\mathrm{Ob}$ die noch vorhandenen festen Fettsäuren in ihrer Gänze als solche aus dem verwendeten Öle stammen oder ob sie wenigstens zum Theile Umwandlungsproducte der Ölsäure sind, lässt sich natürlich nicht feststellen. 